\title{
Parametrized Multilinear Littlewood-Paley Operators on Hardy Spaces
}

\author{
Sha He* and Qingying Xue
}

\begin{abstract}
In this paper, we study the parametrized multilinear Marcinkiewicz integral $\mu^{\rho}$ and the multilinear Littlewood-Paley $g_{\lambda}^{*}$-function. We proved that if the kernel $\Omega$ associated to parametrized multilinear Marcinkiewicz integral $\mu^{\rho}$ is homogeneous of degree zero and satisfies the Lipschitz continuous condition, or the kernel $K$ associated to the multilinear Littlewood-Paley $g_{\lambda}^{*}$-function satisfies the Hörmander condition, then they are bounded from $H^{p_{1}} \times \cdots \times H^{p_{m}}$ to $L^{p}$ with $m n /(m n+\gamma)<p_{1}, \ldots, p_{m} \leq 1$ and $1 / p=1 / p_{1}+\cdots+1 / p_{m}$.
\end{abstract}

\section{Introduction and main results}

In 1960, Hörmander [10] first studied the parametrized Marcinkiewicz integral $\mu^{\rho}$ which is connected closely with the Marcinkiewicz integral defined and studied by Stein 17 in 1958. He proved that $\mu^{\rho}$ is $L^{p}$ bounded if the kernel $\Omega$ satisfies some Dini type conditions. Later, Sakamoto and Yabuta 15 showed that the parametrized Lusin area integral $S^{\rho}$ and the parametrized Littlewood-Paley $g_{\lambda}^{*}$-function are bounded on $L^{p}$ provided the kernel $\Omega$ satisfies the Lipschitz conditions.

It was well known that the multilinear operator was first studied by Coifman and Meyer 4, 5]. After that, the multilinear theory has been paid great attention, and it develops rapidly in recent years. Especially, Christ and Journé [2], Kenig and Stein [11], Grafakos and Torres [9] and Lerner et al. [13 have made great contributions to this field. On the other hand, in 1972, Fefferman and Stein [6] studied the classical CalderónZygmund operators on the real Hardy space $H^{p}\left(\mathbb{R}^{n}\right)(0<p<1)$. Later, Grafakos and Kalton [7] investigated the boundedness of the nonconvolution multilinear CalderónZygmund operators on Hardy spaces. Recently, Li et al. [14] extended the results of [7] to the weighted case. Shi et al. [16 introduced the multilinear Littlewood-Paley $g_{\lambda}^{*}$ function

Received July 10, 2017; Accepted May 20, 2018.

Communicated by Duy-Minh Nhieu.

2010 Mathematics Subject Classification. 42B20, 42B25, 42B30.

Key words and phrases. parametrized multilinear Littlewood-Paley $\mu^{\rho}$-function, multilinear LittlewoodPaley $g_{\lambda}^{*}$-function, Hardy spaces.

This work was partially supported by NSFC (Nos. 11471041, 11671039) and NSFC-DFG (No. 11761131002), the NSF of Zhejiang Province of China (No. LQ17A010002) and the China Postdoctoral Science Foundation (No. 2017M620687).

*Corresponding author. 
with the kernel $K$ satisfies the Hörmander condition and proved the strong weighted estimates and the weak type boundedness of $g_{\lambda}^{*}$.

There is a natural question about multilinear Littlewood-Paley operators, that is, are these operators bounded on Hardy spaces? The aim of this paper is to investigate the boundedness of the multilinear Littlewood-Paley operators on Hardy spaces.

Throughout this paper, for any set $E, \chi_{E}$ will be used to denote the characteristic function of the set $E$. The letter $C$ will always denote a positive constant that may vary at each occurrence but is independent of the essential variable.

In order to state our results, we first introduce some definitions and notations.

Definition 1.1. Let $\Omega$ be a function defined on $\left(\mathbb{R}^{n}\right)^{m}$ with the following properties:

(i) $\Omega$ is homogeneous of degree 0 , i.e.,

$$
\Omega(\lambda y)=\Omega(y)
$$

where $y=\left(y_{1}, \ldots, y_{m}\right) \in\left(\mathbb{R}^{n}\right)^{m}$.

(ii) $\Omega$ is Lipschitz continuous on $\left(S^{n-1}\right)^{m}$, i.e., there are $0<\gamma<1$ and $C>0$ such that for any $\xi=\left(\xi_{1}, \ldots, \xi_{m}\right), \eta=\left(\eta_{1}, \ldots, \eta_{m}\right) \in\left(\mathbb{R}^{n}\right)^{m}$,

$$
|\Omega(\xi)-\Omega(\eta)| \leq C\left|\xi^{\prime}-\eta^{\prime}\right|^{\gamma}
$$

where $y^{\prime}=\left(y_{1}, \ldots, y_{m}\right)^{\prime}=\left(y_{1}, \ldots, y_{m}\right) /\left(\left|y_{1}\right|+\cdots+\left|y_{m}\right|\right)$.

For any $\vec{f}=\left(f_{1}, \ldots, f_{m}\right) \in \mathcal{S}\left(\mathbb{R}^{n}\right) \times \cdots \times \mathcal{S}\left(\mathbb{R}^{n}\right), t>0$, we define

$$
\begin{gathered}
K^{\rho}\left(y_{1}, \ldots, y_{m}\right)=\frac{\Omega\left(y_{1}, \ldots, y_{m}\right) \chi_{(B(0,1))^{m}}\left(y_{1}, \ldots, y_{m}\right)}{\left(\left|y_{1}\right|+\cdots+\left|y_{m}\right|\right)^{m(n-\rho)}} \\
K_{t}^{\rho}\left(y_{1}, \ldots, y_{m}\right)=\frac{1}{t^{m n}} K^{\rho}\left(\frac{y_{1}}{t}, \ldots, \frac{y_{m}}{t}\right)
\end{gathered}
$$

and

$$
\begin{aligned}
G_{t}^{\rho}(\vec{f})(y) & =K_{t}^{\rho} *\left(f_{1} \otimes \cdots \otimes f_{m}\right)(y) \\
& =\frac{1}{t^{m \rho}} \int_{(B(y, t))^{m}} \frac{\Omega\left(y-z_{1}, \ldots, y-z_{m}\right)}{\left(\left|y-z_{1}\right|+\cdots+\left|y-z_{m}\right|\right)^{m(n-\rho)}} \prod_{i=1}^{m} f_{i}\left(z_{i}\right) d z_{i},
\end{aligned}
$$

where $B(x, t)=\left\{y \in \mathbb{R}^{n}:|y-x| \leq t\right\}$.

Then the parametrized multilinear Marcinkiewicz integral $\mu^{\rho}$ is defined by

$$
\mu^{\rho}(\vec{f})(x)=\left(\int_{0}^{\infty}\left|G_{t}^{\rho}(\vec{f})(x)\right|^{2} \frac{d t}{t}\right)^{1 / 2} .
$$


In this paper, we assume that for $1<q_{1}, \ldots, q_{m}<\infty$ satisfying $1 / q=1 / q_{1}+\cdots+1 / q_{m}$, there exists a constant $C>0$ such that

$$
\left\|\mu^{\rho}(\vec{f})\right\|_{L^{q}} \leq C \prod_{i=1}^{m}\left\|f_{i}\right\|_{L^{q_{i}}}
$$

In fact, considering the bilinear case, the parametrized bilinear Marcinkiewicz integral $\mu^{\rho}$-function can be rewritten as a 4-linear Fourier multiplier with symbol

$$
m^{\rho}\left(\xi_{1}, \xi_{2}, \zeta_{1}, \zeta_{2}\right)=\int_{0}^{\infty} \widehat{\psi^{\rho}}\left(t \xi_{1}, t \xi_{2}\right) \widehat{\psi^{\rho}}\left(t \zeta_{1}, t \zeta_{2}\right) \frac{d t}{t}
$$

If assume the kernel is sufficiently smooth, then by the results of Grafakos-Miyachi-Tomita 8, we know that the parametrized bilinear Marcinkiewicz integral $\mu^{\rho}$-function does satisfy the boundedness in (1.3), which shows our assumption $(1.3)$ is reasonable.

The first result in this paper is as follows.

Theorem 1.2. Let $\gamma>0, \rho \in(0,(n+\gamma) / m)$. Suppose $\Omega$ satisfies 1.1 and 1.2), if $f_{i} \in H^{p_{i}}\left(\mathbb{R}^{n}\right)$, where $p_{i} \in(m n /(m n+\gamma), 1]$ for $i=1, \ldots, m$, then for any $p$ with $1 / p=1 / p_{1}+\cdots+1 / p_{m}$, there exists a constant $C>0$ such that

$$
\left\|\mu^{\rho}(\vec{f})\right\|_{L^{p}\left(\mathbb{R}^{n}\right)} \leq C \prod_{i=1}^{m}\left\|f_{i}\right\|_{H^{p_{i}\left(\mathbb{R}^{n}\right)}} .
$$

To obtain the boundedness of multilinear Littlewood-Paley $g_{\lambda}^{*}$ function, we also introduce another kind of multilinear standard kernel, we say a function $K$ defined on $\left(\mathbb{R}^{n}\right)^{m}$ is a multilinear standard kernel, if $K$ satisfies the following two conditions:

$$
\left|K\left(y_{1}, \ldots, y_{m}\right)\right| \leq \frac{C}{\left(1+\sum_{i=1}^{m}\left|y_{i}\right|\right)^{m n+\delta}}
$$

for some $\delta>0$, and all $\left(y_{1}, \ldots, y_{m}\right) \in\left(\mathbb{R}^{n}\right)^{m}$,

$$
\left|K\left(y_{1}, \ldots, y_{i}+z, \ldots, y_{m}\right)-K\left(y_{1}, \ldots, y_{i}, \ldots, y_{m}\right)\right| \leq \frac{C|z|^{\gamma}}{\left(1+\sum_{i=1}^{m}\left|y_{i}\right|\right)^{m n+\delta+\gamma}}
$$

for some $\gamma>0, i=1,2, \ldots, m, 2|z| \leq \max _{i=1, \ldots, m}\left\{\left|y_{i}\right|\right\}$ and all $\left(y_{1}, \ldots, y_{m}\right) \in\left(\mathbb{R}^{n}\right)^{m}$.

For any $\vec{f}=\left(f_{1}, \ldots, f_{m}\right) \in \mathcal{S}\left(\mathbb{R}^{n}\right) \times \cdots \times \mathcal{S}\left(\mathbb{R}^{n}\right), t>0$ and $z \notin \bigcap_{i=1}^{m}$ supp $f_{i}$, we will denote by $G_{t}$ as

$$
G_{t}(\vec{f})(z)=\frac{1}{t^{m n}} \int_{\left(\mathbb{R}^{n}\right)^{m}} K\left(\frac{z-y_{1}}{t}, \ldots, \frac{z-y_{m}}{t}\right) \prod_{i=1}^{m} f_{i}\left(y_{i}\right) d y_{i} .
$$

The multilinear Littlewood-Paley $g_{\lambda}^{*}$ function is defined by

$$
g_{\lambda}^{*}(\vec{f})(x)=\left[\iint_{\mathbb{R}_{+}^{n+1}}\left(\frac{t}{t+|x-z|}\right)^{n \lambda}\left|G_{t}(\vec{f})(z)\right|^{2} \frac{d z d t}{t^{n+1}}\right]^{1 / 2}, \quad \lambda>1 .
$$


We point out that this kind of Littlewood-Paley $g_{\lambda}^{*}$ function was introduced by Shi et al. [16, in which they obtain the strong weighted estimates and the weak type boundedness of $g_{\lambda}^{*}$ for $p_{i} \geq 1$. Our second result is as follows:

Theorem 1.3. Let $\lambda>2 m$ and $\gamma \in(0, n(\lambda-2 m) / 2)$. If $f_{i} \in H^{p_{i}}\left(\mathbb{R}^{n}\right)$, where $p_{i} \in$ $(m n /(m n+\gamma), 1]$ for $i=1, \ldots, m$, then for any $p$ with $1 / p=1 / p_{1}+\cdots+1 / p_{m}$, there exists a constant $C>0$ such that

$$
\left\|g_{\lambda}^{*}(\vec{f})\right\|_{L^{p}\left(\mathbb{R}^{n}\right)} \leq C \prod_{i=1}^{m}\left\|f_{i}\right\|_{H^{p_{i}\left(\mathbb{R}^{n}\right)}} .
$$

Remark 1.4. If we define the parametrized multilinear Littlewood-Paley $g_{\lambda}^{*, \rho}$-function by $\Omega$, our method doesn't work. It leaves open that whether the parametrized multilinear Littlewood-Paley $g_{\lambda}^{*, \rho}$-function is bounded on Hardy spaces.

The article is organized as follows. The proofs of Theorems 1.2 and 1.3 will be shown in Section 2. Throughout this paper, if $f \leq C g$, we denote by $f \lesssim g$ for short.

\section{Proofs of Theorems 1.2 and 1.3}

Proof of Theorem 1.2. We will prove Theorem 1.2 by using the atomic decomposition of $H^{p}$. See Coifman [3] and Latter [12] for detail. At first, we give the definition of atoms in $\mathbb{R}^{n}$.

Definition 2.1. Let $0<p \leq 1,1<q \leq \infty$. A function $a$ is called an $L^{q}$-atom for $H^{p}\left(\mathbb{R}^{n}\right)$ if there exists a cube $Q$ such that

(i) $\operatorname{supp} a \subset Q$,

(ii) $\|a\|_{L^{q}} \leq|Q|^{1 / q-1 / p}$,

(iii) $\int_{Q} a(x) d x=0$.

Latter 12 proved the following atomic decomposition in $\mathbb{R}^{n}$.

Theorem 2.2. [12] Let $0<p \leq 1,1<q \leq \infty$. A distribution $f$ is in $H^{p}\left(\mathbb{R}^{n}\right)$ if and only if there exists a sequence of $L^{q}$ atoms $a_{i}$ for $H^{p}\left(\mathbb{R}^{n}\right)$ and a sequence of non-negative real numbers $\lambda_{i}$ such that

$$
f=\sum_{i=0}^{\infty} \lambda_{i} a_{i}
$$

in the sense of distributions and

$$
A\|f\|_{H^{p}\left(\mathbb{R}^{n}\right)} \leq\left(\sum_{i=0}^{\infty} \lambda_{i}^{p}\right)^{1 / p} \leq B\|f\|_{H^{p}\left(\mathbb{R}^{n}\right)},
$$

where $A, B$ are constants which depend only on $n$ and $p$. 
Since finite sums of atoms are dense in $H^{p}$, we will work with such sums and we will obtain estimates independent of the number of terms in each sum. The general case will follow by a simple density argument. By the atomic decomposition of $H^{p}$, we split each $f_{i}, i=1, \ldots, m$, as a finite sum of $L^{\infty}$-atoms $a_{i, k_{i}}$ for $H^{p_{i}}$. This means there exist cubes $Q_{i, k_{i}}$, such that

$$
\begin{gathered}
\operatorname{supp} a_{i, k_{i}} \subset Q_{i, k_{i}}, \\
\left\|a_{i, k_{i}}\right\|_{L^{\infty}} \leq\left|Q_{i, k_{i}}\right|^{-1 / p_{i}}, \\
\int_{Q_{i, k_{i}}} a_{i, k_{i}}(x) d x=0 .
\end{gathered}
$$

For a cube $Q$, let $\widetilde{Q}$ be the cube with the same center as $Q$ and $8 \sqrt{n}$ its side length. Using multilinearity, we write

$$
\mu^{\rho}\left(f_{1}, \ldots, f_{m}\right)(x) \leq \sum_{k_{1}, \ldots, k_{m}} \lambda_{1, k_{1}} \cdots \lambda_{m, k_{m}} \mu^{\rho}\left(a_{1, k_{1}}, \ldots, a_{m, k_{m}}\right)(x)
$$

Set

$$
\left|\mu^{\rho}\left(f_{1}, \ldots, f_{m}\right)(x)\right| \leq I_{1}+I_{2}
$$

where

$$
\begin{aligned}
& I_{1}=\sum_{k_{1}, \ldots, k_{m}}\left|\lambda_{1, k_{1}}\right| \cdots\left|\lambda_{m, k_{m}}\right|\left|\mu^{\rho}\left(a_{1, k_{1}}, \ldots, a_{m, k_{m}}\right)(x)\right| \chi_{\widetilde{Q}_{1, k_{1}} \cap \cdots \cap \widetilde{Q}_{m, k_{m}}}, \\
& I_{2}=\sum_{k_{1}, \ldots, k_{m}}\left|\lambda_{1, k_{1}}\right| \cdots\left|\lambda_{m, k_{m}}\right|\left|\mu^{\rho}\left(a_{1, k_{1}}, \ldots, a_{m, k_{m}}\right)(x)\right| \chi_{\widetilde{Q}_{1, k_{1}}^{c} \cup \cdots \cup \widetilde{Q}_{m, k_{m}}^{c}} .
\end{aligned}
$$

Now, let us begin to discuss $I_{1}$. For fixed $k_{1}, \ldots, k_{m}$, assume that

$$
\widetilde{Q}_{1, k_{1}} \cap \cdots \cap \widetilde{Q}_{m, k_{m}} \neq \emptyset
$$

since otherwise there is nothing needs to be proved.

Suppose that $Q_{1, k_{1}}$ has the smallest size among all these cubes. We take a cube $G_{k_{1}, \ldots, k_{m}}$ such that

$$
\widetilde{Q}_{1, k_{1}} \cap \cdots \cap \widetilde{Q}_{m, k_{m}} \subset G_{k_{1}, \ldots, k_{m}} \subset \widetilde{G}_{k_{1}, \ldots, k_{m}} \subset \widetilde{\widetilde{Q}}_{1, k_{1}} \cap \cdots \cap \widetilde{\widetilde{Q}}_{m, k_{m}}
$$

and

$$
\left|G_{k_{1}, \ldots, k_{m}}\right| \geq C\left|Q_{1, k_{1}}\right| .
$$

By Hölder's inequality and [1, Theorem 1.2], we have

$$
\frac{1}{\left.\mid G_{k_{1}, \ldots, k_{m}}\right)} \int_{G_{k_{1}, \ldots, k_{m}}}\left|\mu^{\rho}\left(a_{1, k_{1}}, \ldots, a_{m, k_{m}}\right)(x)\right| d x
$$




$$
\begin{aligned}
& \lesssim\left|G_{k_{1}, \ldots, k_{m}}\right|^{-1 / 2}\left\|\mu^{\rho}\left(a_{1, k_{1}}, \ldots, a_{m, k_{m}}\right)\right\|_{L^{2}} \\
& \lesssim\left|G_{k_{1}, \ldots, k_{m}}\right|^{-1 / 2}\left\|a_{1, k_{1}}\right\|_{L^{2}} \prod_{i=2}^{m}\left\|a_{i, k_{i}}\right\|_{L^{\infty}} \\
& \lesssim\left|G_{k_{1}, \ldots, k_{m}}\right|^{-1 / 2}\left|Q_{1, k_{1}}\right|^{1 / 2-1 / p_{1}} \prod_{i=2}^{m}\left|Q_{i, k_{i}}\right|^{-1 / p_{i}} \\
& \lesssim\left(\prod_{i=1}^{m}\left|Q_{i, k_{i}}\right|^{-1 / p_{i}}\right) .
\end{aligned}
$$

Then we need the following lemma.

Lemma 2.3. [7] Let $0<p \leq 1$. Then there is a constant $C=C(p, n)$ such that for all finite collection $\left\{Q_{k}\right\}_{k=1}^{m}$ of cubes in $\mathbb{R}^{n}$ and all nonnegative functions $g_{k} \in L^{p}$ with $\operatorname{supp} g_{k} \subset Q_{k}$ we have

$$
\left\|\sum_{k=1}^{m} g_{k}\right\|_{L^{p}} \leq C\left\|\sum_{k=1}^{m}\left(\frac{1}{\left|Q_{k}\right|} \int_{Q_{k}} g_{k}(x) d x\right) \chi_{\widetilde{Q}_{k}}\right\|_{L^{p}} .
$$

By Lemma 2.3 and Hölder's inequality, we have

$$
\begin{aligned}
\left\|I_{1}\right\|_{L^{p}} & \lesssim\left\|\sum_{k_{1}, \ldots, k_{m}}\left|\lambda_{1, k_{1}}\right| \cdots\left|\lambda_{m, k_{m}}\right| \prod_{i=1}^{m}\left|Q_{i, k_{i}}\right|^{-1 / p_{i}} \chi_{\widetilde{\widetilde{Q}}_{1, k_{1}}} \cdots \chi_{\widetilde{\widetilde{Q}}_{m, k m}}\right\|_{L^{p}} \\
& \lesssim\left\|\prod_{i=1}^{m}\left(\sum_{k_{i}}\left|\lambda_{i, k_{i}}\right|\left|Q_{i, k_{i}}\right|^{-1 / p_{i}} \chi_{\widetilde{\widetilde{Q}}_{i, k_{i}}}\right)\right\|_{L^{p}} \\
& \lesssim \prod_{i=1}^{m}\left\|\left(\sum_{k_{i}}\left|\lambda_{i, k_{i}}\right|\left|Q_{i, k_{i}}\right|^{-1 / p_{i}} \chi_{\widetilde{\widetilde{Q}}_{i, k_{i}}}\right)\right\|_{L^{p_{i}}} \\
& \lesssim \prod_{i=1}^{m}\left(\sum_{k_{i}}\left|\lambda_{i, k_{i}}\right|^{p_{i}}\left|Q_{i, k_{i}}\right|^{-1}\left|\widetilde{\widetilde{Q}}_{i, k_{i}}\right|\right) \\
& \lesssim \prod_{i=1}^{m}\left(\sum_{k_{i}}\left|\lambda_{i, k_{i}}\right|^{p_{i}}\right)^{1 / p_{i}} \\
& \lesssim \prod_{i=1}^{m}\left\|f_{i}\right\|_{H^{p_{i}}} .
\end{aligned}
$$

Then, we estimate $I_{2}(x)$. Let $A$ be a nonempty subset of $\{1, \ldots, m\}$, and we denote the cardinality of $A$ by $|A|$. Then $1 \leq|A| \leq m$. Set $A^{c}=\{1, \ldots, m\} \backslash A$. For $A=\{1, \ldots, m\}$, we define

$$
\left(\bigcap_{i \in A} \widetilde{Q}_{i, k_{i}}^{c}\right) \cap\left(\bigcap_{i \in A^{c}} \widetilde{Q}_{i, k_{i}}\right)=\bigcap_{i \in A} \widetilde{Q}_{i, k_{i}}^{c}
$$


Then we have

$$
\widetilde{Q}_{1, k_{1}}^{c} \cup \cdots \cup \widetilde{Q}_{m, k_{m}}^{c}=\bigcup_{A \subset\{1, \ldots, m\}}\left(\left(\bigcap_{i \in A} \widetilde{Q}_{i, k_{i}}^{c}\right) \cap\left(\bigcap_{i \in A^{c}} \widetilde{Q}_{i, k_{i}}\right)\right) .
$$

For fixed $1 \leq r \leq m$, without loss of generality, we consider the particular case, that is, by permuting the indices, we assume $x \in E_{r}$, where

$$
E_{r}=\left(\widetilde{Q}_{1, k_{1}}^{c} \cap \cdots \cap \widetilde{Q}_{r, k_{r}}^{c}\right) \cap\left(\widetilde{Q}_{r+1, k_{r+1}} \cap \cdots \cap \widetilde{Q}_{m, k_{m}}\right) .
$$

Denoting the center of $Q_{i, k_{i}}$ by $c_{i, k_{i}}$ and the sidelength of $Q_{i, k_{i}}$ by $l_{i, k_{i}}$, let us estimate $I_{2}$. For fixed $E_{r}, x \in E_{r}, y_{i} \in Q_{i, k_{i}}, 1 \leq i \leq r$, we have

$$
\left|x-y_{i}\right| \sim\left|x-c_{i, k_{i}}\right|
$$

There exists $i_{0} \in\{1, \ldots, r\}$ such that

$$
\left|x-c_{i_{0}, k_{i_{0}}}\right|=\max \left\{\left|x-c_{i, k_{i}}\right|: i \in\{1, \ldots, r\}\right\} .
$$

Then, if $t<\left|x-c_{i_{0}, k_{i_{0}}}\right|-l_{i_{0}, k_{i_{0}}}$, then $B(x, t) \cap Q_{i_{0}, k_{i_{0}}}=\emptyset$. Thus, using the vanish condition of atom, we have

$$
\begin{aligned}
& \left|\mu^{\rho}\left(a_{1, k_{1}}, \ldots, a_{m, k_{m}}\right)(x)\right| \\
& =\left[\int_{0}^{\infty} \mid \int_{(B(x, t))^{m}} \frac{\Omega\left(x-y_{1}, \ldots, x-y_{m}\right)}{\left(\sum_{i=1}^{m}\left|x-y_{i}\right|\right)^{m(n-\rho)}}\right. \\
& \left.\times\left. a_{1, k_{1}}\left(y_{1}\right) \cdots a_{m, k_{m}}\left(y_{m}\right) d y_{1} \cdots d y_{m}\right|^{2} \frac{d t}{t^{1+2 m \rho}}\right]^{1 / 2} \\
& =\left[\int_{\left|x-c_{i_{0}, k_{i}}\right|-l_{i_{0}, k_{i}}}^{\infty} \mid \int_{(B(x, t))^{m-r}} \int_{\mathbb{R}^{r n}} \frac{\Omega\left(x-y_{1}, \ldots, x-y_{m}\right)}{\left(\sum_{i=1}^{r}\left|x-c_{i, k_{i}}\right|+\sum_{i=r+1}^{m}\left|x-y_{i}\right|\right)^{m(n-\rho)}}\right. \\
& \left.\times\left. a_{1, k_{1}}\left(y_{1}\right) \cdots a_{m, k_{m}}\left(y_{m}\right) d y_{1} \cdots d y_{m}\right|^{2} \frac{d t}{t^{1+2 m \rho}}\right]^{1 / 2} \\
& =\left[\int_{\left|x-c_{i_{0}, k_{i}}\right|-l_{i_{0}, k_{i}}}^{\infty} \mid \int_{(B(x, t))^{m-r}} \int_{\mathbb{R}^{r n}}\right. \\
& \times \frac{\Omega\left(x-y_{1}, \ldots, x-y_{m}\right)-\Omega\left(x-c_{1, k_{1}}, \ldots, x-y_{m}\right)}{\left(\sum_{i=1}^{r}\left|x-c_{i, k_{i}}\right|+\sum_{i=r+1}^{m}\left|x-y_{i}\right|\right)^{m(n-\rho)}} \\
& \left.\times\left. a_{1, k_{1}}\left(y_{1}\right) \cdots a_{m, k_{m}}\left(y_{m}\right) d y_{1} \cdots d y_{m}\right|^{2} \frac{d t}{t^{1+2 m \rho}}\right]^{1 / 2} \\
& \lesssim \prod_{i=1}^{m}\left|Q_{i, k_{i}}\right|^{-1 / p_{i}}\left[\int _ { | x - c _ { i _ { 0 } , k _ { i } } | - l _ { i _ { 0 } , k _ { i } } } ^ { \infty } \left(\int_{(B(x, t))^{m-r}} \int_{Q_{1, k_{1}} \times \cdots \times Q_{r, k_{r}}}\right.\right. \\
& \left.\left.\times \frac{\left|\Omega\left(x-y_{1}, \ldots, x-y_{m}\right)-\Omega\left(x-c_{1, k_{1}}, \ldots, x-y_{m}\right)\right|}{\left(\sum_{i=1}^{r}\left|x-c_{i, k_{i}}\right|+\sum_{i=r+1}^{m}\left|x-y_{i}\right|\right)^{m(n-\rho)}} d y_{1} \cdots d y_{m}\right)^{2} \frac{d t}{t^{1+2 m \rho}}\right]^{1 / 2} .
\end{aligned}
$$


From $(1.2)$ and the fact that $Q_{1, k_{1}}$ is the smallest cube among all the cubes, we see that

$$
\begin{aligned}
& \left|\Omega\left(x-y_{1}, \ldots, x-y_{m}\right)-\Omega\left(x-c_{1, k_{1}}, \ldots, x-y_{m}\right)\right| \\
\lesssim & \left|\frac{\left(x-y_{1}, \ldots, x-y_{m}\right)}{\sum_{i=1}^{m}\left|x-y_{i}\right|}-\frac{\left(x-c_{1, k_{1}}, \ldots, x-y_{m}\right)}{\left|x-c_{1, k_{1}}\right|+\sum_{i=2}^{m}\left|x-y_{i}\right|}\right|^{\gamma} \\
\lesssim & \frac{l_{1, k_{1}}^{\gamma}}{\left(\sum_{i=1}^{m}\left|x-y_{i}\right|\right)^{\gamma}} .
\end{aligned}
$$

Thus,

$$
\begin{aligned}
& \left|\mu^{\rho}\left(a_{1, k_{1}}, \ldots, a_{m, k_{m}}\right)(x)\right| \\
\lesssim & \prod_{i=1}^{m}\left|Q_{i, k_{i}}\right|^{-1 / p_{i}}\left[\int _ { | x - c _ { i _ { 0 } , k _ { i } } | - l _ { i _ { 0 } , k _ { i } } } ^ { \infty } \left(\int_{\left(\mathbb{R}^{n}\right)^{m-r}} \int_{Q_{1, k_{1}} \times \cdots \times Q_{r, k_{r}}}\right.\right. \\
& \left.\left.\times \frac{l_{1, k_{1}}^{\gamma}}{\left(\sum_{i=1}^{r}\left|x-c_{i, k_{i}}\right|+\sum_{i=r+1}^{m}\left|x-y_{i}\right|\right)^{m(n-\rho)+\gamma}} d y_{1} \cdots d y_{m}\right)^{2} \frac{d t}{t^{1+2 m \rho}}\right]^{1 / 2} \\
\lesssim & \prod_{i=1}^{r}\left|Q_{i, k_{i}}\right|^{1-1 / p_{i}} \prod_{i=r+1}^{m}\left|Q_{i, k_{i}}\right|^{-1 / p_{i}} \\
& \times \int_{\left(\mathbb{R}^{n}\right)^{m-r}} \frac{l_{1, k_{1}}^{\gamma}}{\left(\sum_{i=1}^{r}\left|x-c_{i, k_{i}}\right|+\sum_{i=r+1}^{m}\left|x-y_{i}\right|\right)^{m(n-\rho)+\gamma}} d y_{r+1} \cdots d y_{m} \\
& \left.\times \int_{\left|x-c_{i_{0}, k_{i}}\right|-l_{i_{0}, k_{i}}}^{\infty} \frac{d t}{t^{1+2 m \rho}}\right]^{1 / 2} \\
\lesssim & \prod_{i=1}^{r}\left|Q_{i, k_{i}}\right|^{1-1 / p_{i}} \prod_{i=r}^{m}\left|Q_{i, k_{i}}\right|^{-1 / p_{i}} \frac{l_{1, k_{1}}^{\gamma}}{\left(\sum_{i=1}^{r}\left|x-c_{i, k_{i}}\right|\right)^{r n+\gamma}} \\
\lesssim & \left.\prod_{i=1}^{r} \frac{l_{i, k_{i}}^{n-n / p_{i}+\gamma / r}}{\left(\left|x-c_{i, k_{i}}\right|+l_{i, k_{i}}\right)^{n+\gamma / r}} \prod_{i=r+1}^{m} l_{i, k_{i}}\right|^{-n / p_{i}} \\
\lesssim & \prod_{i=1}^{m-n / p_{i}+\gamma / r} \frac{l_{i, k_{i}}}{\left(\left|x-c_{i, k_{i}}\right|+l_{i, k_{i}}\right)^{n+\gamma / r}}
\end{aligned}
$$

where we use

$$
\int_{\mathbb{R}^{n}} \frac{1}{(b+|y-c|)^{M}} d y \leq b^{n-M}
$$

if $M>n, b$ is any positive number and $c \in \mathbb{R}^{n}$ in the third inequality.

Summing over all possible $1 \leq r \leq m$ and all possible combinations of subset of $\{1, \ldots, m\}$ of size $r$ we obtain the pointwise estimate

$$
\left|\mu^{\rho}\left(a_{1, k_{1}}, \ldots, a_{m, k_{m}}\right)(x)\right| \lesssim \prod_{i=1}^{m} \frac{l_{i, k_{i}}^{n-n / p_{i}+\gamma / m}}{\left(\left|x-c_{i, k_{i}}\right|+l_{i, k_{i}}\right)^{n+\gamma / m}}
$$


for all $x \in \widetilde{Q}_{1, k_{1}}^{c} \cup \cdots \cup \widetilde{Q}_{m, k_{m}}^{c}$.

Hence, by the fact $p_{i}>m n /(m n+\gamma)$, we see that

$$
\begin{aligned}
\left\|I_{2}\right\|_{L^{p}} & \lesssim\left\|\sum_{k_{1}, \ldots, k_{m}}\left|\lambda_{1, k_{1}}\right| \cdots\left|\lambda_{m, k_{m}}\right| \prod_{i=1}^{m} \frac{l_{i, k_{i}}^{n-n / p_{i}+\gamma / m}}{\left(\left|x-c_{i, k_{i}}\right|+l_{i, k_{i}}\right)^{n+\gamma / m}}\right\|_{L^{p}} \\
& \lesssim \prod_{i=1}^{m} \|\left.\sum_{k_{i}}\left|\lambda_{i, k_{i}}\right| \frac{l_{i, k_{i}}^{n-n / p_{i}+\gamma / m}}{\left(\left|x-c_{i, k_{i}}\right|+l_{i, k_{i}}\right)^{n+\gamma / m}}\right|_{L^{p_{i}}} \\
& \lesssim \prod_{i=1}^{m}\left\{\int_{\mathbb{R}^{n}}\left|\sum_{k_{i}}\right| \lambda_{i, k_{i}}\left|\frac{l_{i, k_{i}}^{n-n / p_{i}+\gamma / m}}{\left(\left|x-c_{i, k_{i}}\right|+l_{i, k_{i}}\right)^{n+\gamma / m}}\right|^{p_{i}} d x\right\}^{1 / p_{i}} \\
& \lesssim \prod_{i=1}^{m}\left\{\sum_{k_{i}}\left|\lambda_{i, k_{i}}\right|^{p_{i}} l_{i, k_{i}}^{n p_{i}-n+\gamma p_{i} / m} \int_{\mathbb{R}^{n}} \frac{1}{\left(\left|x-c_{i, k_{i}}\right|+l_{i, k_{i}}\right)^{n p_{i}+\gamma p_{i} / m}} d x\right\}^{m} \\
& \lesssim \prod_{i=1}^{m}\left\{\sum_{k_{i}}\left|\lambda_{i, k_{i}}\right|^{p_{i}}\right\}^{1 / p_{i}} \\
& \lesssim \prod_{i=1}^{m}\left\|f_{i}\right\|_{H^{p_{i}}},
\end{aligned}
$$

which combined with 2.2, completes the proof of Theorem 1.2 .

Proof of Theorems 1.3. Use the same notations as in the proof of Theorems 1.2. Similar to the proof of Theorems 1.2 , it suffices to show that, for all $x \in \widetilde{Q}_{1, k_{1}}^{c} \cup \cdots \cup \widetilde{Q}_{m, k_{m}}^{c}$,

$$
\left|g_{\lambda}^{*}\left(a_{1, k_{1}}, \ldots, a_{m, k_{m}}\right)(x)\right| \lesssim \prod_{i=1}^{m} \frac{l_{i, k_{i}}^{n-n / p_{i}+\gamma / m}}{\left(\left|x-c_{i, k_{i}}\right|+l_{i, k_{i}}\right)^{n+\gamma / m}} .
$$

Assume $x \in E_{r}$, where

$$
E_{r}=\left(\widetilde{Q}_{1, k_{1}}^{c} \cap \cdots \cap \widetilde{Q}_{r, k_{r}}^{c}\right) \cap\left(\widetilde{Q}_{r+1, k_{r+1}} \cap \cdots \cap \widetilde{Q}_{m, k_{m}}\right),
$$

and assume the side length of the cube $Q_{1, k_{1}}$ is the smallest among the side length of the cube $Q_{i, k_{i}}, 1 \leq i \leq r$.

Denoting the center of $Q_{i, k_{i}}$ by $c_{i, k_{i}}$ and the sidelength of $Q_{i, k_{i}}$ by $l_{i, k_{i}}$, let us estimate $I_{2}$. For fixed $E_{r}, x \in E_{r}, y_{i} \in Q_{i, k_{i}}, 1 \leq i \leq r$, we have

$$
\left|x-y_{i}\right| \sim\left|x-c_{i, k_{i}}\right|
$$

There exists $i_{0} \in\{1, \ldots, r\}$ such that

$$
\left|x-c_{i_{0}, k_{i_{0}}}\right|=\max \left\{\left|x-c_{i, k_{i}}\right|: i \in\{1, \ldots, r\}\right\} .
$$


For $x \in E_{r}$ and $z \in \mathbb{R}^{n}$, let

$$
A(x, z):=\left\{\left(y_{1}, \ldots, y_{m}\right) \in\left(\mathbb{R}^{n}\right)^{m}: 2\left|y_{1}-c_{1, k_{1}}\right| \leq \max _{1 \leq i \leq m}\left|x+z-y_{i}\right|\right\} .
$$

Thus, using the vanish condition of atom, we have

$$
\begin{aligned}
& \left|g_{\lambda}^{*}\left(a_{1, k_{1}}, \ldots, a_{m, k_{m}}\right)(x)\right| \\
& =\left\{\int_{0}^{\infty} \int_{\mathbb{R}^{n}}\left(\frac{t}{t+|z|}\right)^{n \lambda} \mid \int_{\left(\mathbb{R}^{n}\right)^{m}}\right. \\
& \times\left[K\left(\frac{x+z-y_{1}}{t}, \ldots, \frac{x+z-y_{m}}{t}\right)-K\left(\frac{x+z-c_{1, k_{1}}}{t}, \ldots, \frac{x+z-y_{m}}{t}\right)\right] \\
& \left.\times\left. a_{1, k_{1}}\left(y_{1}\right) \cdots a_{m, k_{m}}\left(y_{m}\right) d y_{1} \cdots d y_{m}\right|^{2} \frac{d z d t}{t^{2 m n+n+1}}\right\}^{1 / 2} \\
& \leq\left\{\int_{0}^{\infty} \int_{\mathbb{R}^{n}}\left(\frac{t}{t+|z|}\right)^{n \lambda} \mid \int_{A}\right. \\
& \times\left[K\left(\frac{x+z-y_{1}}{t}, \ldots, \frac{x+z-y_{m}}{t}\right)-K\left(\frac{x+z-c_{1, k_{1}}}{t}, \ldots, \frac{x+z-y_{m}}{t}\right)\right] \\
& \left.\times\left. a_{1, k_{1}}\left(y_{1}\right) \cdots a_{m, k_{m}}\left(y_{m}\right) d y_{1} \cdots d y_{m}\right|^{2} \frac{d z d t}{t^{2 m n+n+1}}\right\}^{1 / 2} \\
& +\left\{\int_{0}^{\infty} \int_{\mathbb{R}^{n}}\left(\frac{t}{t+|z|}\right)^{n \lambda} \mid \int_{A^{c}}\right. \\
& \times\left[K\left(\frac{x+z-y_{1}}{t}, \ldots, \frac{x+z-y_{m}}{t}\right)-K\left(\frac{x+z-c_{1, k_{1}}}{t}, \ldots, \frac{x+z-y_{m}}{t}\right)\right] \\
& \left.\times\left. a_{1, k_{1}}\left(y_{1}\right) \cdots a_{m, k_{m}}\left(y_{m}\right) d y_{1} \cdots d y_{m}\right|^{2} \frac{d z d t}{t^{2 m n+n+1}}\right\}^{1 / 2} \\
& =: \text { I }+ \text { II. }
\end{aligned}
$$

From 1.5, we see that

$$
\begin{aligned}
\mathrm{I} \lesssim & \left\{\int_{0}^{\infty} \int_{\mathbb{R}^{n}}\left(\frac{t}{t+|z|}\right)^{n \lambda} \mid \int_{\left(\mathbb{R}^{n}\right)^{m}} \frac{\left|y_{1}-c_{1, k_{1}}\right|^{\gamma} / t^{\gamma}}{\left(1+\sum_{i=1}^{m} \frac{\left|x+z-y_{i}\right|}{t}\right)^{m n+\delta+\gamma}}\right. \\
& \left.\times\left. a_{1, k_{1}}\left(y_{1}\right) \cdots a_{m, k_{m}}\left(y_{m}\right) d y_{1} \cdots d y_{m}\right|^{2} \frac{d z d t}{t^{2 m n+n+1}}\right\}^{1 / 2} \\
\lesssim & \left\{\int_{0}^{\infty} \int_{\mathbb{R}^{n}}\left(\frac{t}{t+|z|}\right)^{n \lambda} \mid \int_{\left(\mathbb{R}^{n}\right)^{m}} \frac{\left|y_{1}-c_{1, k_{1}}\right|^{\gamma}}{\left(t+\sum_{i=1}^{m}\left|x+z-y_{i}\right|\right)^{m n+\delta+\gamma}}\right. \\
& \left.\times\left. a_{1, k_{1}}\left(y_{1}\right) \cdots a_{m, k_{m}}\left(y_{m}\right) d y_{1} \cdots d y_{m}\right|^{2} \frac{d z d t}{t^{n+1-2 \delta}}\right\}^{1 / 2},
\end{aligned}
$$

which, together with, 2.1 and the fact

$$
\int_{\mathbb{R}^{n}} \frac{1}{(b+|y-c|)^{M}} d y \leq b^{n-M},
$$


further implies that

$$
\begin{aligned}
\mathrm{I} \lesssim l_{1, k_{1}}^{\gamma} & \prod_{i=1}^{m} l_{i, k_{i}}^{-n / p_{i}}\left\{\int_{0}^{\infty} \int_{\mathbb{R}^{n}}\left(\frac{t}{t+|z|}\right)^{n \lambda}\right. \\
\times & \left.\left|\int_{Q_{1, k_{1}} \times \cdots \times Q_{r, k_{r}}} \frac{1}{\left(t+\sum_{i=1}^{r}\left|x+z-y_{i}\right|\right)^{n r+\delta+\gamma}} d y_{1} \cdots d y_{r}\right|^{2} \frac{d z d t}{t^{n+1-2 \delta}}\right\}^{1 / 2} \\
\lesssim & l_{1, k_{1}}^{\gamma} \prod_{i=1}^{m} l_{i, k_{i}}^{-n / p_{i}}\left\{\int_{0}^{\left|x-c_{i_{0}, k_{i}}\right|} \int_{\mathbb{R}^{n}}\left(\frac{t}{t+|z|}\right)^{n \lambda}\right. \\
& \left.\times\left|\int_{Q_{1, k_{1}} \times \cdots \times Q_{r, k_{r}}} \frac{1}{\left(t+\sum_{i=1}^{r}\left|x+z-y_{i}\right|\right)^{n r+\delta+\gamma}} d y_{1} \cdots d y_{r}\right|^{2} \frac{d z d t}{t^{n+1-2 \delta}}\right\}^{1 / 2} \\
+ & l_{1, k_{1}}^{\gamma} \prod_{i=1}^{m} l_{i, k_{i}}^{-n / p_{i}}\left\{\int_{\left|x-c_{i_{0}, k_{i}}\right|}^{\infty} \int_{\mathbb{R}^{n}}\left(\frac{t}{t+|z|}\right)^{n \lambda}\right. \\
& \left.\times\left|\int_{Q_{1, k_{1}} \times \cdots \times Q_{r, k_{r}}} \frac{1}{\left(t+\sum_{i=1}^{r}\left|x+z-y_{i}\right|\right)^{n r+\delta+\gamma}} d y_{1} \cdots d y_{r}\right|^{2} \frac{d z d t}{t^{n+1-2 \delta}}\right\}^{1 / 2} \\
= & l_{1, k_{1}}^{\gamma} \prod_{i=1}^{m} l_{i, k_{i}}^{-n / p_{i}}\left(\mathrm{I}_{1}+\mathrm{I}_{2}\right) .
\end{aligned}
$$

To show

$$
\mathrm{I} \lesssim \prod_{i=1}^{m} \frac{l_{i, k_{i}}^{n-n / p_{i}+\gamma / m}}{\left(\left|x-c_{i, k_{i}}\right|+l_{i, k_{i}}\right)^{n+\gamma / m}}
$$

by the fact that $Q_{1, k_{1}}$ is the smallest cube among $\left\{Q_{i, k_{i}}\right\}, 1 \leq i \leq r$, it suffices to show that

$$
\mathrm{I}_{1}+\mathrm{I}_{2} \lesssim \frac{\prod_{i=1}^{r} l_{i, k_{i}}^{n}}{\left(\left|x-c_{i_{0}, k_{i_{0}}}\right|\right)^{n r+\gamma}}
$$

Let

$$
\begin{gathered}
\Theta_{1}:=\left\{z \in \mathbb{R}^{n}:|z| \leq \frac{1}{2}\left|x-c_{i_{0}, k_{i_{0}}}\right|\right\} \\
\Theta_{2}:=\left\{z \in \mathbb{R}^{n}: \frac{1}{2}\left|x-c_{i_{0}, k_{i_{0}}}\right|<|z|<4\left|x-c_{i_{0}, k_{i_{0}}}\right|\right\}
\end{gathered}
$$

and

$$
\Theta_{3}:=\left\{z \in \mathbb{R}^{n}:|z| \geq 4\left|x-c_{i_{0}, k_{i_{0}}}\right|\right\} .
$$

When $t \in\left(0,\left|x-c_{i_{0}, k_{i_{0}}}\right|\right)$ and $z \in \Theta_{1} \cup \Theta_{3}$, we have

$$
t+\sum_{i=1}^{r}\left|x+z-y_{i}\right| \gtrsim \sum_{i=1}^{r}\left|x+z-y_{i}\right| \gtrsim\left|x-c_{i_{0}, k_{i}}\right| .
$$


Thus,

$$
\begin{aligned}
& \left\{\int_{0}^{\left|x-c_{i_{0}, k_{i}}\right|} \int_{\Theta_{1} \cup \Theta_{3}}\left(\frac{t}{t+|z|}\right)^{n \lambda}\right. \\
& \left.\times\left|\int_{Q_{1, k_{1}} \times \cdots \times Q_{r, k_{r}}} \frac{1}{\left(t+\sum_{i=1}^{r}\left|x+z-y_{i}\right|\right)^{n r+\delta+\gamma}} d y_{1} \cdots d y_{r}\right|^{2} \frac{d z d t}{t^{n+1-2 \delta}}\right\}^{1 / 2} \\
\lesssim & \frac{\prod_{i=1}^{r} l_{i, k_{i}}^{n}}{\left(\left|x-c_{i_{0}, k_{i}}\right|\right)^{n r+\gamma+\delta}}\left\{\int_{0}^{\left|x-c_{i_{0}, k_{i}}\right|} \int_{\Theta_{1} \cup \Theta_{3}}\left(\frac{t}{t+|z|}\right)^{n \lambda} \frac{d z d t}{t^{n+1-2 \delta}}\right\}^{1 / 2} \\
\lesssim & \frac{\prod_{i=1}^{r} l_{i, k_{i}}^{n}}{\left(\left|x-c_{i_{0}, k_{i_{0}}}\right|\right)^{n r+\gamma+\delta}}\left\{\int_{0}^{\left|x-c_{i_{0}, k_{i}}\right|} \frac{d t}{t^{1-2 \delta}}\right\}^{1 / 2} \\
\lesssim & \frac{\prod_{i=1}^{r} l_{i, k_{i}}^{n}}{\left(\left|x-c_{i_{0}, k_{i}}\right|\right)^{n r+\gamma}} .
\end{aligned}
$$

On the other hand, since $\gamma<\frac{n}{2}(\lambda-2 m)$, there exists some $\epsilon>0$ such that $n(\lambda-$ $2 m)-2 \gamma-2 \epsilon>0$, thus

$$
\begin{aligned}
& \left\{\int_{0}^{\left|x-c_{i_{0}, k_{i_{0}}}\right|} \int_{\Theta_{2}}\left(\frac{t}{t+|z|}\right)^{n \lambda}\right. \\
& \left.\times\left|\int_{Q_{1, k_{1}} \times \cdots \times Q_{r, k_{r}}} \frac{1}{\left(t+\sum_{i=1}^{r}\left|x+z-y_{i}\right|\right)^{n r+\delta+\gamma}} d y_{1} \cdots d y_{r}\right|^{2} \frac{d z d t}{t^{n+1-2 \delta}}\right\}^{1 / 2} \\
\lesssim & \prod_{i=1, i \neq i_{0}}^{r} l_{i, k_{i}}^{n}\left\{\int_{0}^{\left|x-c_{i_{0}, k_{i_{0}}}\right|} \int_{\Theta_{2}}\left(\frac{t}{\left|x-c_{i_{0}, k_{0}}\right|}\right)^{n \lambda}\right. \\
& \left.\times\left|\int_{Q_{i_{0}, k_{i}}} \frac{1}{t^{n(r-1 / 2)+\delta+\gamma+\epsilon}} \frac{1}{\left|x+z-y_{i_{0}}\right|^{n / 2-\epsilon}} d y_{i_{0}}\right|^{2} \frac{d z d t}{t^{n+1-2 \delta}}\right\}^{1 / 2} \\
& \frac{\prod_{i=1}^{r} l_{i, k_{i}}^{n}}{\left(\left|x-c_{i_{0}, k_{i_{0}}}\right|\right)^{n \lambda / 2}}\left\{\int_{0}^{\left|x-c_{i_{0}, k_{i}}\right|} \frac{t^{n \lambda}}{t^{2 n r+2 \gamma+2 \epsilon+1}} d t\right\}^{1 / 2} \\
& \times\left\{\int_{\Theta_{2}}\left|\int_{Q_{i_{0}, k_{i}}} \frac{1}{\left.\left|x+z-y_{i_{0}}\right|\right|^{n / 2-\epsilon}} d y_{i_{0}}\right|^{2} d z\right\}^{1 / 2} \\
\lesssim & \frac{\prod_{i=1}^{r} l_{i, k_{i}}^{n}}{\left(\left|x-c_{i_{0}, k_{i_{0}}}\right|\right)^{n r+\gamma}} .
\end{aligned}
$$

When $t \in\left[\left|x-c_{i_{0}, k_{i_{0}}}\right|, \infty\right)$, we have

$$
t+\sum_{i=1}^{r}\left|x+z-y_{i}\right| \gtrsim t .
$$


Thus,

$$
\begin{aligned}
& \left\{\int_{\left|x-c_{i_{0}, k_{0}}\right|}^{\infty} \int_{\mathbb{R}^{n}}\left(\frac{t}{t+|z|}\right)^{n \lambda}\right. \\
& \left.\quad \times\left|\int_{Q_{1, k_{1}} \times \cdots \times Q_{r, k_{r}}} \frac{1}{\left(t+\sum_{i=1}^{r}\left|x+z-y_{i}\right|\right)^{n r+\delta+\gamma}} d y_{1} \cdots d y_{r}\right|^{2} \frac{d z d t}{t^{n+1-2 \delta}}\right\}^{1 / 2} \\
& \lesssim \prod_{i=1}^{r} l_{i, k_{i}}^{n}\left\{\int_{\mid x-c_{i_{0}, k_{i}}}^{\infty} \int_{\mathbb{R}^{n}}\left(\frac{t}{t+|z|}\right)^{n \lambda} \frac{d z d t}{t^{2 n r+2 \gamma+n+1}}\right\}^{1 / 2} \\
& \lesssim \frac{\prod_{i=1}^{r} l_{i, k_{i}}^{n}}{\left(\left|x-c_{i_{0}, k_{i}}\right|\right)^{n r+\gamma}},
\end{aligned}
$$

where we use

$$
\int_{\mathbb{R}^{n}}\left(\frac{t}{t+|z|}\right)^{n \lambda} d z \lesssim t^{n}
$$

as $\lambda>1$.

Combining (2.6), 2.7) and (2.8), we show that (2.5) holds and hence (2.4).

Now we estimate II. From (1.4), and $2 l_{1, k_{1}} \geq 2\left|y_{1}-c_{1, k_{1}}\right| \geq \max _{1 \leq i \leq m}\left|x+z-y_{i}\right|$, we see that

$$
\begin{aligned}
\mathrm{II} \lesssim & \left\{\int_{0}^{\infty} \int_{\mathbb{R}^{n}}\left(\frac{t}{t+|z|}\right)^{n \lambda} \mid \int_{\left(\mathbb{R}^{n}\right)^{m}} \frac{l_{1, k_{1}}^{\gamma}}{\left(1+\sum_{i=1}^{m} \frac{\left|x+z-y_{i}\right|}{t}\right)^{m n+\delta}\left(\sum_{i=1}^{m}\left|x+z-y_{i}\right|\right)^{\gamma}}\right. \\
& \left.\times\left. a_{1, k_{1}}\left(y_{1}\right) \cdots a_{m, k_{m}}\left(y_{m}\right) d y_{1} \cdots d y_{m}\right|^{2} \frac{d z d t}{t^{2 m n+n+1}}\right\}^{1 / 2} \cdot
\end{aligned}
$$

Then, by the same method as in the proof of I, we have

$$
\mathrm{II} \lesssim \prod_{i=1}^{m} \frac{l_{i, k_{i}}^{n-n / p_{i}+\gamma / m}}{\left(\left|x-c_{i, k_{i}}\right|+l_{i, k_{i}}\right)^{n+\gamma / m}}
$$

which, together with (2.4), further completes the proof of (2.3) and hence Theorem 1.3 .

\section{Acknowledgments}

The authors thank the referee(s) to read the manuscript carefully and make some nice suggestions. Also, the authors want to thank Dr. Jingquan Yan for discussion in the revised version.

\section{References}

[1] X. Chen, Q. Xue and K. Yabuta, On multilinear Littlewood-Paley operators, Nonlinear Anal. 115 (2015), 25-40. 
[2] M. Christ and J.-L. Journé, Polynomial growth estimates for multilinear singular integral operators, Acta Math. 159 (1987), no. 1-2, 51-80.

[3] R. R. Coifman, A real variable characterization of $H^{p}$, Studia Math. 51 (1974), 269274.

[4] R. R. Coifman and Y. Meyer, On commutators of singular integrals and bilinear singular integrals, Trans. Amer. Math. Soc. 212 (1975), 315-331.

[5] _ Commutateurs d'intégrales singulières et opérateurs multilin'eaires, Ann. Inst. Fourier (Grenoble) $\mathbf{2 8}$ (1978), no. 3, 177-202.

[6] C. Fefferman and E. M. Stein, $H^{p}$ spaces of several variables, Acta Math. 129 (1972), no. 3-4, 137-193.

[7] L. Grafakos and N. Kalton, Multilinear Calderón-Zygmund operators on Hardy spaces, Collect. Math. 52 (2001), no. 2, 169-179.

[8] L. Grafakos, A. Miyachi and N. Tomita, On multilinear Fourier multipliers of limited smoothness, Canad. J. Math. 65 (2013), no. 2, 299-330.

[9] L. Grafakos and R. H. Torres, Multilinear Calderón-Zygmund theory, Adv. Math. 165 (2002), no. 1, 124-164.

[10] L. Hörmander, Estimates for translation invariant operators in $L^{p}$ spaces, Acta Math. 104 (1960), 93-140.

[11] C. E. Kenig and E. M. Stein, Multilinear estimates and fractional integration, Math. Res. Lett. 6 (1999), no. 1, 1-15.

[12] R. H. Latter, A characterization of $H^{p}\left(\mathbb{R}^{n}\right)$ in terms of atoms, Studia Math. 62 (1978), no. 1, 92-101.

[13] A. K. Lerner, S. Ombrosi, C. Pérez, R. H. Torres and R. Trujillo-González, New maximal functions and multiple weights for the multilinear Calderón-Zygmund theory, Adv. Math. 220 (2009), no. 4, 1222-1264.

[14] W. Li, Q. Xue and K. Yabuta, Multilinear Calderón-Zygmund operators on weighted Hardy spaces, Studia Math. 199 (2010), no. 1, 1-16.

[15] M. Sakamoto and K. Yabuta, Boundedness of Marcinkiewicz functions, Studia Math. 135 (1999), no. 2, 103-142.

[16] S. Shi, Q. Xue and K. Yabuta, On the boundedness of multilinear Littlewood-Paley $g_{\lambda}^{*}$ function, J. Math. Pures Appl. 101 (2014), no. 3, 394-413. 
[17] E. M. Stein, On the functions of Littlewood-Paley, Lusin, and Marcinkiewicz, Trans. Amer. Math. Soc. 88 (1958), 430-466.

Sha He

Institute of Applied Physics and Computational Mathematics, Beijing, 100088,

P. R. China

E-mail address: amyhesha@163.com

Qingying Xue

School of Mathematical Sciences, Beijing Normal University, Laboratory of Mathematics and Complex Systems, Ministry of Education, Beijing, 100875, P. R. China

E-mail address: qyxue@bnu.edu.cn 